\title{
KONTSEVICH QUANTIZATION AND INVARIANT DISTRIBUTIONS ON LIE GROUPS
}

\author{
BY MARTIN ANDLER, ALEXANDER DVORSKY AND \\ SIDDHARTHA SAHI
}

Dedicated to Israel M. Gelfand

ABSTRACT. - We study Kontsevich's deformation quantization for the dual of a finite-dimensional real Lie algebra (or superalgebra) $\mathfrak{g}$. In this case the Kontsevich $\star$-product defines a new convolution on $S(\mathfrak{g})$, regarded as the space of distributions supported at $0 \in \mathfrak{g}$. For $p \in S(\mathfrak{g})$, we show that the convolution operator $f \longmapsto p \star f$ is a differential operator with analytic germ.

We use this fact to prove a conjecture of Kashiwara and Vergne on invariant distributions on a Lie group $G$. This implies local solvability of bi-invariant differential operators on a Lie supergroup. In the special case of Lie groups, we get a new proof Duflo's theorem.

(C) 2002 Éditions scientifiques et médicales Elsevier SAS

RÉSUMÉ. - Nous étudions la quantification par déformation de Kontsevich du dual d'une algèbre (ou superalgèbre) de Lie réelle de dimension finie $\mathfrak{g}$. Dans ce cas, le $\star$-produit de Kontsevich définit une nouvelle convolution sur $S(\mathfrak{g})$, vu comme l'espace des distributions de support le point $0 \in \mathfrak{g}$. Pour $p \in S(\mathfrak{g})$, nous démontrons que l'opérateur de convolution $f \longmapsto p \star f$ est un opérateur différentiel de germe analytique.

Nous utilisons ce fait pour prouver une conjecture de Kashiwara et Vergne sur les distributions invariantes sur un groupe de Lie $G$. Ceci implique la résolubilité locale des opérateurs différentiels bi-invariants sur un super-groupe de Lie $G$. Dans le cas particulier des groups, nous obtenons ainsi une nouvelle démonstration du théorème de Duflo.

(c) 2002 Éditions scientifiques et médicales Elsevier SAS

\section{Introduction}

In recent work [1], M. Kontsevich has established a remarkable result on the formality of the Hochschild complex of a smooth manifold. An important consequence of this result is an explicit construction of an associative $\star$-product on an arbitrary smooth Poisson manifold $(X, \gamma)$. This $\star$-product is given by a formal power series of bidifferential operators (depending on a parameter $\hbar$ ).

For a general Poisson manifold this series does not converge, except for $\hbar=0$. Thus the analytic properties of the $\star$-product are somewhat obscure. However, as we shall show in this paper, if $X=\mathfrak{g}^{*}$ is the dual of a finite-dimensional real Lie algebra $\mathfrak{g}$ (or, in general, if the coefficients of the Poisson bracket $\gamma$ are linear), then the situation is much nicer. 
In this case, we can set $\hbar=1$, and Kontsevich's $\star$-product descends to an actual product on the algebra of polynomial functions on $\mathfrak{g}^{*}$, which can be naturally identified with the symmetric algebra $\mathcal{S}=\mathcal{S}(\mathfrak{g})$. In this paper, we will identify $\mathcal{S}(\mathfrak{g})$ with the convolution algebra of distributions on $\mathfrak{g}$ supported at 0 . Then the $\star$-product can be viewed as a new associative convolution product of such distributions.

Let $\mathfrak{D}$ be the algebra of germs at 0 of differential operators on $\mathfrak{g}$ with analytic coefficients. By virtue of the pairing between functions and distributions, $\mathcal{S}$ is naturally a right $\mathfrak{D}$-module and to emphasize this fact we shall write the action on distributions on the right. Our first main result is the following

THEOREM 0.1. - Given $p \in \mathcal{S}$ of order l, there is a unique element $\partial_{p}^{\star}$ of order l in $\mathfrak{D}$ such that for all $r \in \mathcal{S}$

$$
r \star p=r \cdot \partial_{p}^{\star} .
$$

Next, in $[1,8.3 .3]$, Kontsevich introduces a certain formal power series

$$
S_{1}(x)=\exp \left(\sum_{k=1}^{\infty} c_{2 k}^{(1)} \operatorname{tr}\left[(\operatorname{ad} x)^{2 k}\right]\right),
$$

where the constants $c_{2 k}^{(1)}$ are expressed as integrals of smooth differential forms over certain manifolds with corners (compactified configuration spaces, introduced in [12]).

Proposition 0.2. - The power series $S_{1}(x)$ converges to an analytic function $\tau(x)$ in some neighborhood of 0 in $\mathfrak{g}$.

For $a \in \mathfrak{g}$, define the adjoint vector field $\operatorname{adj}_{a}$ by the formula

$$
\operatorname{adj}_{a}(f)(x)=\left.\frac{d}{d t} f(\exp (-t a) \cdot x)\right|_{t=0} .
$$

Let

$$
\mathcal{I}=\mathcal{S}^{\mathfrak{g}}=\left\{p \in \mathcal{S} \mid p \cdot \operatorname{adj}_{a}=0 \text { for all } a \in \mathfrak{g}\right\}
$$

be the subalgebra of invariant distributions, and let $\mathfrak{R}$ be the right ideal of $\mathfrak{D}$ generated by the germs of the differential operators $\operatorname{adj}_{a}, a \in \mathfrak{g}$.

Our next result is the following

THEOREM 0.3. - For $p \in \mathcal{I}$, the operator

$$
T_{p}=\partial_{p} \tau-\tau \partial_{p \tau}^{\star}
$$

\section{belongs to $\Re$.}

Here $p \tau \in \mathcal{S}$ and $\partial_{p \tau}^{\star}$ is the corresponding differential operator defined in Theorem 0.1 , and for $r \in \mathcal{S}, r \cdot \partial_{p}=r *_{\mathfrak{g}} p$ (convolution of distributions on $\mathfrak{g}$ ).

We now discuss some applications of these results to invariant distributions on Lie groups. Let $G$ be a finite-dimensional Lie group with Lie algebra $\mathfrak{g}$. We write $\mathcal{U}=\mathcal{U}(\mathfrak{g})$ for the enveloping algebra of $\mathfrak{g}$, and $\mathcal{Z}=\mathcal{U}^{\mathfrak{g}}$ for the center of the enveloping algebra. We identify $\mathcal{U}$ with the convolution algebra of distributions on $G$ supported at $1 \in G$.

Let $\mathbf{U}$ and $\mathbf{S}$ be the spaces of germs at 1 and 0 of distributions on $G$ and $\mathfrak{g}$, and let $\mathbf{Z}$ and $\mathbf{I}$ be the subspaces of $\mathfrak{g}$-invariant germs. We denote by $\exp _{*}: \mathbf{S} \rightarrow \mathbf{U}$ and $\exp ^{*}: \mathbf{U} \rightarrow \mathbf{S}$ 
the pushforward and pullback of distributions (germs) under the exponential map; and define $\eta: \mathbf{S} \rightarrow \mathbf{U}$ by

$$
\eta(p)=\exp _{*}(p q)
$$

where for $x \in \mathfrak{g}$

$$
q(x):=\operatorname{det}\left(\frac{\mathrm{e}^{\operatorname{ad}(x) / 2}-\mathrm{e}^{-\operatorname{ad}(x) / 2}}{\operatorname{ad}(x)}\right)^{1 / 2}=\exp \left(\sum_{k=1}^{\infty} \frac{B_{2 k}}{4 k(2 k) !} \operatorname{tr}\left[(\operatorname{ad} x)^{2 k}\right]\right) .
$$

Here $B_{2 k}$ are the Bernoulli numbers.

It follows from [1, Ch. 8], that the restriction of $\eta$ to $\mathcal{I}$ is an algebra isomorphism from $\mathcal{I}$ to $\mathcal{Z}$, i.e.,

$$
\eta\left(p_{1} *_{\mathfrak{g}} p_{2}\right)=\eta\left(p_{1}\right) *_{G} \eta\left(p_{2}\right)
$$

for $p_{1}, p_{2} \in \mathcal{I}$, where $*_{\mathfrak{g}}$ and $*_{G}$ denote convolutions in the Lie algebra and Lie group respectively. (This fact was first established by Duflo in [8-10] using Kirillov's orbit method. See also [6] for an overview of Duflo's construction.)

From Theorems 0.1 and 0.3 , we can deduce

THEOREM 0.4. - For $p \in \mathcal{I}$, the following differential operator $D_{p}: \mathbf{S} \rightarrow \mathbf{S}$ lies in $\mathfrak{R}:$

$$
P \cdot D_{p}=\exp ^{*}\left(\eta\left(P *_{\mathfrak{g}} p\right)-\eta(P) *_{G} \eta(p)\right) .
$$

This result was conjectured by M. Kashiwara and M. Vergne in [14], and proved by them for $\mathfrak{g}$ solvable. It has several important corollaries. First, since operators from $\mathfrak{R}$ annihilate distributions from $\mathbf{I}$, we obtain the following extension of the isomorphism (2):

TheOREM 0.5. - For $p \in \mathcal{I}$ and $P \in \mathbf{I}$, we have $\eta\left(P *_{\mathfrak{g}} p\right)=\eta(P) *_{G} \eta(p)$.

Next, recall that a distribution $Q$ on $G$ is called an eigendistribution if there is a character $\chi: \mathcal{Z} \longrightarrow \mathbb{C}$ such that

$$
Q *_{G} z=\chi(z) Q \text { for all } z \text { in } \mathcal{Z} .
$$

Eigendistributions on the Lie algebra are defined similarly, and we obtain the following

THEOREM 0.6. - The map $\eta$ takes germs of invariant eigendistributions on the Lie algebra to those on the Lie group.

As another consequence of Theorem 0.5, we obtain

THEOREM 0.7. - Every nonzero bi-invariant differential operator on $G$ admits a (local) fundamental solution.

Note that the analogous statement for left-invariant differential operators on $G$ is known to be false. In fact, the first example of a not locally solvable partial differential operator [18] is a left-invariant vector field on a three-dimensional Heisenberg group.

COROLlary 0.8. - Every nonzero bi-invariant differential operator on $G$ is locally solvable.

This last result was originally proved by Duflo in [10] (see also [13,20,11] for various special cases). An interpretation of the statement of Theorem 0.7 in terms of Kirillov's orbit method can be found in [15, 6.1 and 8.2]. 
Most of our results extend to the case of finite-dimensional Lie superalgebras without much additional effort. For simplicity of exposition, we work with ordinary Lie algebras and at the end explain the modifications necessary for the super-setting.

The results of this paper were announced in [2].

Added in proof. A recent e-print of B. Shoikhet [22] shows that the function $\tau(x)$ is identically 1 . Incorporating this fact would somewhat simplify the presentation below, but will not substantially change the arguments or yield a sharper result.

\section{Relevant facts from [1]}

\subsection{Configuration spaces}

Following Kontsevich, for $2 n+m \geqslant 2$, we define

$$
\operatorname{Conf}_{n, m}=\left\{\left(p_{1}, \ldots, p_{n} ; q_{1}, \ldots, q_{m}\right) \mid p_{i} \in \mathcal{H}, p_{i_{1}} \neq p_{i_{2}}, q_{j} \in \mathbb{R}, q_{j_{1}} \neq q_{j_{2}}\right\} .
$$

Here $\mathcal{H}=\{z \in \mathbb{C}: \operatorname{Im} z>0\}$.

Conf $_{n, m}$ is a smooth oriented manifold of dimension $2 n+m$, with a free action of the twodimensional group $G^{1}=\{z \rightarrow a z+b \mid a>0, b \in \mathbb{R}\}$. The quotient space

$$
C_{n, m}=\operatorname{Conf}_{n, m} / G^{1}
$$

is a smooth oriented manifold of dimension $2 n+m-2$.

The spaces Conf ${ }_{n, m}$ and $C_{n, m}$ have $m$ ! connected components, and we denote by Conf $_{n, m}^{+}$ and $C_{n, m}^{+}$the component where

$$
q_{1}<q_{2}<\cdots<q_{m}
$$

Similarly, for $n \geqslant 2$, we define

$$
\begin{aligned}
\operatorname{Conf}_{n} & =\left\{\left(p_{1}, \ldots, p_{n}\right) \mid p_{i} \in \mathbb{C}, p_{i_{1}} \neq p_{i_{2}}\right\} \quad \text { and } \\
C_{n} & =\operatorname{Conf}_{n} / G^{2}, \quad \text { where } G^{2}=\{z \rightarrow a z+b \mid a>0, b \in \mathbb{C}\} .
\end{aligned}
$$

Clearly, $\operatorname{dim} C_{n}=2 n-3$.

Consider, for example, the space

$$
C_{2,0}=\left\{\left(p_{1}, p_{2}\right) \in \mathcal{H}^{2} \mid p_{1} \neq p_{2}\right\} / G^{1} .
$$

For each point $c \in C_{2,0}$ we can choose a unique representative of the form $(\sqrt{-1}, z) \in \operatorname{Conf}_{2,0}$. Thus $C_{2,0}$ is homeomorphic to $\mathcal{H} \backslash\{\sqrt{-1}\}$.

Similarly, it is easy to see that

$$
\begin{aligned}
C_{2} & \approx S^{1}, \\
C_{1,1} & \approx(0,1), \\
C_{0,2} & \approx\{0,1\} .
\end{aligned}
$$

The spaces $C_{n, m}$ have natural compactifications $\bar{C}_{n, m}$, such that the boundary components in $\bar{C}_{n, m} \backslash C_{n, m}$ correspond to various degenerations of the configurations of points. 


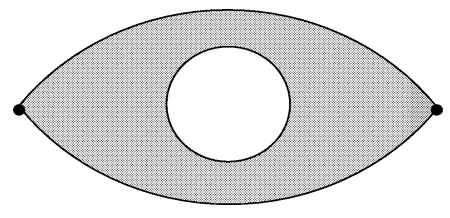

Fig. 1. $\bar{C}_{2,0}$ (THE EYE).

For example,

$$
\begin{aligned}
\bar{C}_{2} & =C_{2}, \\
\bar{C}_{1,1} & =C_{1,1} \sqcup C_{0,2}=[0,1] .
\end{aligned}
$$

Of particular interest is the space

$$
\bar{C}_{2,0}=C_{2,0} \sqcup\left(C_{0,2} \sqcup C_{1,1} \sqcup C_{1,1} \sqcup C_{2}\right),
$$

which can be drawn as "THE EYE".

The circle $C_{2}$ represents two points coming close together in the interior of $\mathcal{H}$, and two arcs $C_{1,1} \sqcup C_{1,1}$ represent the first point (or the second point) coming close to the real line. Finally, the two corners $C_{0,2}$ correspond to both points approaching the real line.

The compactification $\bar{C}_{n, m}$ is described in general in [1, Ch. 5], and the boundary components are products of various $C_{k, l}$ 's and $C_{p}$ 's.

In particular, the boundary components of codimension 1 in $\bar{C}_{n, m}$ are of the form

1. $C_{k} \times C_{n-k+1, m}(k \geqslant 2)$ or

2. $C_{k, l} \times C_{n-k, m-l+1}(k \geqslant 1$ or $l \geqslant 2)$.

The first case corresponds to a cluster of $k$ points in $\mathcal{H}$ coming infinitely close to a point in $\mathcal{H}$, and the second case corresponds to a cluster of $k$ points in $\mathcal{H}$ and $l$ points in $\mathbb{R}$ coming infinitely close to a point in $\mathbb{R}$.

\subsection{Graphs and weights}

We consider the class of "admissible" oriented labeled graphs $\left\{\Gamma=\left(V_{\Gamma}, E_{\Gamma}\right)\right\}$ such that

- $V_{\Gamma}$ consists of $n$ vertices of the "first" type labeled $\{1, \ldots, n\}$ and $m$ vertices of the "second" type labeled $\{\overline{1}, \overline{2}, \ldots, \bar{m}\}$.

- For each vertex $i$ of the first type, there are $k_{i}$ edges in $E_{\Gamma}$ starting from $i$, labeled

$$
\left\{e_{i}^{1}, e_{i}^{2}, \ldots, e_{i}^{k_{i}}\right\}
$$

- $E_{\Gamma}$ contains no edges starting from the vertices of second type, and no loops (edges of type $(v, v))$ or multiple edges.

For each point in $\left(p_{1}, \ldots, p_{n} ; q_{1}, \ldots, q_{m}\right) \in \operatorname{Conf}_{n, m}$, we can draw the graph $\Gamma$ in the closed upper half-plane $\overline{\mathcal{H}}=\mathcal{H} \sqcup \mathbb{R}$ as follows:

vertices $i$ are placed at $p_{i} \in \mathcal{H}$, vertices $\bar{j}$ are placed at $q_{j} \in \mathbb{R}$, and edges are drawn as Lobachevsky geodesics (semicircles centered on $\mathbb{R}$ ) connecting the vertices.

For each edge $e \in E_{\Gamma}$ connecting the vertex at $z_{1} \in \mathcal{H}$ to $z_{2} \in \overline{\mathcal{H}}$, we define $\phi_{e}$ to be the angle between $e$ and the vertical line through $z_{1}$. Then

$$
\phi_{e}=\frac{1}{2 \sqrt{-1}} \log \frac{\left(z_{2}-z_{1}\right)\left(\bar{z}_{2}-z_{1}\right)}{\left(z_{2}-\bar{z}_{1}\right)\left(\bar{z}_{2}-\bar{z}_{1}\right)} .
$$


Define a map $\Phi_{\Gamma}$ from $\operatorname{Conf}_{n, m}$ to the $k$-dimensional torus $\mathbb{T}^{k}$, where $k=\sum_{i=1}^{n} k_{i}$ as follows

$$
\Phi_{\Gamma}\left(p_{1}, \ldots, p_{n} ; q_{1}, \ldots, q_{m}\right)=\left(\phi_{e_{1}^{1}}, \phi_{e_{1}^{2}}, \ldots, \phi_{e_{n}^{k_{n}}}\right) .
$$

Observe that $\Phi_{\Gamma}$ descends to a map from $C_{n, m}$ to $\mathbb{T}^{k}$. Define $\omega_{\Gamma}$ to be the pullback to $C_{n, m}$ of the normalized volume form (total measure 1 ) on $\mathbb{T}^{k}$.

The form $\omega_{\Gamma}$ is a smooth $k$-form on $C_{n, m}$, which extends continuously to $\bar{C}_{n, m}$. We define the weight ${ }^{1} w_{\Gamma}$ by

$$
w_{\Gamma}=\int_{\bar{C}_{n, m}^{+}} \omega_{\Gamma} .
$$

Evidently, $w_{\Gamma}=0$, unless $k=\operatorname{dim} C_{n, m}=2 n+m-2$.

\subsection{Star-product}

Let

$$
\gamma\left(f_{1}, f_{2}\right)=\sum_{i, j=1}^{d} \gamma^{i j} \partial_{i}\left(f_{1}\right) \partial_{j}\left(f_{2}\right)
$$

be a smooth Poisson bracket (bivector field) on $C^{\infty}\left(\mathbb{R}^{d}\right)$. Kontsevich's main result gives the following construction of an associative star-product on $C^{\infty}\left(\mathbb{R}^{d}\right)[[h]]$ :

$$
f_{1} \star f_{2}=\sum_{n=0}^{\infty} \frac{\hbar^{n}}{n !} B_{n}\left(f_{1}, f_{2}\right),
$$

where $B_{0}\left(f_{1}, f_{2}\right)=f_{1} f_{2}, B_{1}\left(f_{1}, f_{2}\right)=\gamma\left(f_{1}, f_{2}\right)$ and in general

$$
B_{n}\left(f_{1}, f_{2}\right)=\sum_{\Gamma \in G_{n}} w_{\Gamma} B_{\Gamma}\left(f_{1}, f_{2}\right) .
$$

Here $G_{n}$ is defined as a class of admissible graphs with $n$ vertices of the first type, two vertices of the second type and $k_{i}=2,1 \leqslant i \leqslant n$. The weight $w_{\Gamma}$ is as in the previous subsection, and $B_{\Gamma}$ is a bidifferential operator defined as follows:

$$
\begin{aligned}
B_{\Gamma}\left(f_{1}, f_{2}\right)= & \sum_{I: E_{\Gamma} \rightarrow\{1,2, \ldots, d\}}\left[\prod_{k=1}^{n}\left(\prod_{e \in E_{\Gamma}, e=(*, k)} \partial_{I(e)}\right) \gamma^{\left.I\left(e_{k}^{1}\right) I\left(e_{k}^{2}\right)\right]}\right. \\
& \times\left(\prod_{e \in E_{\Gamma}, e=(*, \overline{1})} \partial_{I(e)}\right) f_{1} \times\left(\prod_{e \in E_{\Gamma}, e=(*, \overline{2})} \partial_{I(e)}\right) f_{2} .
\end{aligned}
$$

To obtain this operator, we relabel each vertex $k, 1 \leqslant k \leqslant n$, by a component $\gamma^{i_{k} j_{k}}$ of $\gamma$, relabel $\overline{1}$ as $f_{1}$ and $\overline{2}$ as $f_{2}$. We also label the edge $e_{k}^{1}$ with $i_{k}$ and the edge $e_{k}^{2}$ with $j_{k}$. Then an edge leading to the vertex indicates differentiation of the function labeling the vertex with respect to the index labeling the edge.

\footnotetext{
${ }^{1}$ This weight differs from $w_{\Gamma}$ on p. 6 of [1] by the factor of $\frac{1}{n !}$ and from the weight $W_{\Gamma}$ on p. 23 by a factor of $\frac{1}{k_{1} ! \cdots k_{n} !}$.

$4^{\mathrm{e}}$ SÉRIE - TOME $35-2002-\mathrm{N}^{\circ} 3$
} 
Below is an example of a graph from $\Gamma_{0} \in G_{2}$ with

$$
e_{1}^{1}=(1, \overline{1}), \quad e_{1}^{2}=(1,2), \quad e_{2}^{1}=(2, \overline{1}), \quad e_{2}^{2}=(2, \overline{2}) .
$$

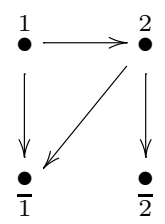

or (relabeling)

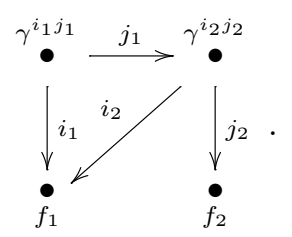

The corresponding bidifferential operator $B_{\Gamma_{0}}$ is given by

$$
B_{\Gamma_{0}}\left(f_{1}, f_{2}\right)=\sum_{1 \leqslant i_{1}, j_{1}, i_{2}, j_{2} \leqslant d} \gamma^{i_{1} j_{1}} \partial_{j_{1}}\left(\gamma^{i_{2} j_{2}}\right) \partial_{i_{1}} \partial_{i_{2}}\left(f_{1}\right) \partial_{j_{2}}\left(f_{2}\right) .
$$

\subsection{Tangent map}

Let $\gamma=\sum_{i, j=1}^{d} \gamma^{i j} \partial_{i} \wedge \partial_{j}$ be a Poisson bivector field on $\mathbb{R}^{d}$ as before; following [1, Ch. 8], we define the map $I_{T}$ from $C^{\infty}\left(\mathbb{R}^{d}\right)$ to $C^{\infty}\left(\mathbb{R}^{d}\right)[[h]]$ by

$$
I_{T}(f)=\sum_{n=0}^{\infty} \frac{\hbar^{n}}{n !} \sum_{\Gamma \in H_{n}} w_{\Gamma} D_{\Gamma}(f)
$$

Here $H_{n}$ is the set of all admissible graphs with $n+1$ vertices of first type and no vertices of the second type such that $k_{i}=2,1 \leqslant i \leqslant n$, and $k_{n+1}=0$. The differential operator $D_{\Gamma}$ is defined by labeling the vertex $n+1$ with $f$, the remaining vertices by components of $\gamma$, and then proceeding as in formula (8).

Let $\mathcal{I}$ be the center of the Poisson algebra $\left(C^{\infty}\left(\mathbb{R}^{d}\right), \gamma\right)$, i.e.

$$
\mathcal{I}=\left\{f \in C^{\infty}\left(\mathbb{R}^{d}\right): \gamma(f, g)=0 \text { for all } g \in C^{\infty}\left(\mathbb{R}^{d}\right)\right\} .
$$

Then, as established in [1, Ch. 8],

$$
I_{T}\left(f_{1}\right) \star I_{T}\left(f_{2}\right)=I_{T}\left(f_{1} f_{2}\right)
$$

for $f_{1}, f_{2} \in \mathcal{I}$.

Let us briefly explain the ideas underlying the above equality.

Kontsevich's main result is a construction of an $L^{\infty}$-quasiisomorphism $\mathcal{U}$ between the differential graded Lie algebras $\mathcal{T}$ and $\mathcal{D}$ consisting of the skew polyvector fields and polydifferential operators on $\mathbb{R}^{d}$, respectively. The quasiisomorphism $\mathcal{U}$ can be regarded as a (geometric) map between formal manifolds ( $Q$-manifolds) associated to $\mathcal{T}$ and $\mathcal{D}$, respectively.

The skew bivector field $\hbar \gamma$ can be regarded as a point distribution $\delta_{\hbar \gamma}$ in the first $Q$-manifold. Then the tangent spaces at $\hbar \gamma$ and $\mathcal{U}(\hbar \gamma)$ are differential graded Lie algebras $\mathcal{T}^{\prime}$ and $\mathcal{D}^{\prime}$, with the same underlying Lie algebras as $\mathcal{T}$ and $\mathcal{D}$, but with modified differentials. The derivative $d \mathcal{U}$ of $\mathcal{U}$ at $\hbar \gamma$ induces a graded linear map between $\mathcal{T}^{\prime}$ and $\mathcal{D}^{\prime}$, and $I_{T}$ is precisely the restriction of $d \mathcal{U}$ to the 0 -vector fields (i.e., functions).

The equality (10) follows from compatibility of $d \mathcal{U}$ with the natural cup product structures on the (tangent) cohomology of $\mathcal{T}^{\prime}$ and $\mathcal{D}^{\prime}$, as established in [1, Theorem 8.2]. 
The argument proving [1, Theorem 8.2], which was merely outlined in [1], was communicated to us by M. Kontsevich [16], and actually proves a slightly stronger result. Since we need this stronger version, we shall reproduce Kontsevich's argument for the case of Lie algebras in Section 3.

\section{Star-product for Lie algebras}

In the rest of the paper we restrict ourselves to the situation when $X=\mathfrak{g}^{*}$ is the dual of a finite dimensional Lie algebra $\mathfrak{g}$, and $\gamma$ is the standard Poisson bracket on $\mathfrak{g}^{*}$. In this case the coefficients of a bivector field $\gamma$ are linear functions on $\mathfrak{g}^{*}$. More precisely, let $x_{1}, x_{2}, \ldots, x_{d}$ be some basis of $\mathfrak{g}$ viewed as coordinate functions on $\mathfrak{g}^{*}$, and let $\left\{c_{i j}^{k}\right\}$ be the corresponding set of structure constants.

We normalize $x_{1}, x_{2}, \ldots, x_{d}$ such that

$$
\left|c_{i j}^{k}\right| \leqslant 2 \text { for all } i, j \text { and } k \text {. }
$$

Then

$$
\left[x_{i}, x_{j}\right]=\sum_{k=1}^{d} c_{i j}^{k} x_{k}
$$

and

$$
\gamma\left(f_{1}, f_{2}\right)=\frac{1}{2} \sum_{i, j, k} c_{i j}^{k} x_{k} \frac{\partial f_{1}}{\partial x_{i}} \frac{\partial f_{2}}{\partial x_{j}},
$$

i.e.,

$$
\gamma^{i j}=\frac{1}{2} \sum_{k=1}^{d} c_{i j}^{k} x_{k} .
$$

The linearity of the coefficients of $\gamma$ implies that the formula (7) for the bidifferential operator $B_{n}$ can be rewritten as

$$
B_{n}=\sum_{\Gamma \in A_{n}} w_{\Gamma} B_{\Gamma}
$$

where $A_{n}$ consists of those graphs in $G_{n}$ for which there is at most one incoming edge at every vertex of the first type. Indeed, if $\Gamma \in G_{n} \backslash A_{n}$, the corresponding bidifferential operator $B_{\Gamma, \gamma}$ is automatically 0 .

Next, let $f_{1}$ and $f_{2}$ be two polynomials on $\mathfrak{g}^{*}$ with $\operatorname{deg}\left(f_{1}\right)=l_{1}, \operatorname{deg}\left(f_{2}\right)=l_{2}$. Then we remark that the graphs contributing to the star-product formula for $f_{1} \star f_{2}$ can have no more than $l_{1}+l_{2}$ vertices of the first type. Indeed, for any $\Gamma \in A_{n}$ the corresponding bidifferential operator $B_{\Gamma, \gamma}$ contains exactly $2 n$ differentiations. When $2 n>n+l_{1}+l_{2}, B_{\Gamma}$ is obviously 0 (because $f_{1}$ can be differentiated at most $l_{1}$ times, $f_{2}$ at most $l_{2}$ times and each of the coefficients of $\gamma$ corresponding to the remaining vertices at most once). Hence

$$
f_{1} \star f_{2}=\sum_{n=0}^{l_{1}+l_{2}} \frac{\hbar^{n}}{n !} \sum_{\Gamma \in A_{n}} w_{\Gamma} B_{\Gamma}\left(f_{1}, f_{2}\right) .
$$


This sum is finite, and if we set $\hbar=1$, we obtain a polynomial on $\mathfrak{g}^{*}$ of degree $l_{1}+l_{2}$. Therefore, Kontsevich's $\star$-product descends to an actual product on the algebra of polynomial functions on $\mathfrak{g}^{*}$, which can be naturally identified with the symmetric algebra $\mathcal{S}=\mathcal{S}(\mathfrak{g})$.

Let $\mathfrak{W}(\mathfrak{g})$ and $\mathfrak{W}\left(\mathfrak{g}^{*}\right)$ be the Weyl algebras of polynomial coefficient differential operators on $\mathfrak{g}$ and $\mathfrak{g}^{*}$ respectively. Both of these algebras are generated by $\mathfrak{g} \oplus \mathfrak{g}^{*}$, and there is a unique antiisomorphism $\iota$ between them, which is the identity on the generators. Observe that $\iota$ interchanges multiplication and differentiation operators.

We will now change our point of view and regard $\mathcal{S}$ as the algebra of distributions with point support $($ at $0 \in \mathfrak{g})$. Then $\mathcal{S}$ is naturally a right module for the algebra $\mathfrak{W}(\mathfrak{g})$ via $\iota$. As discussed in the introduction, we shall write differential operators acting on distributions, on the right.

This approach leads to the following

LemMa 2.1. - Let $p \in \mathcal{S}$ be a homogeneous distribution of order $l$, and let $\Gamma \in A_{n}$. Then there is a differential operator $\partial_{\Gamma}^{p} \in \mathfrak{W}(\mathfrak{g})$ of order at most $l$, with polynomial coefficients of degree at most $n$, such that for any $r \in \mathcal{S}$

$$
B_{\Gamma}(r, p)=r \cdot \partial_{\Gamma}^{p}
$$

Proof. - From the formula (8) for $B_{\Gamma}(r, p)$, it is clear that $B_{\Gamma}(\cdot, p)$ is a differential operator from $\mathfrak{W}\left(\mathfrak{g}^{*}\right)$. Since at most $n$ edges of $\Gamma$ can terminate at the vertex $\overline{1}$ (corresponding to $r$ ), the order of $B_{\Gamma}(\cdot, p)$ is at most $n$. Also, the degree of the coefficients of this differential operator is

$$
n+l-\text { \#edges terminating at vertices other than } \overline{1},
$$

which is at most $l$.

Now let $\partial_{\Gamma}^{p}=\iota\left(B_{\Gamma}(\cdot, p)\right)$. Then the statement follows from the discussion above.

Remark. - Let $y_{1}, \ldots, y_{d}$ be the basis of $\mathfrak{g}^{*}$ dual to $x_{1}, \ldots, x_{d}$, viewed as coordinate functions on $\mathfrak{g}$. Then, by the lemma above, for $p$ homogeneous of order $l$, we can write

$$
r \cdot \partial_{\Gamma}^{p}=r \cdot\left(\sum_{|\beta|=l}\left(\sum_{\alpha} c_{\alpha \beta}^{\Gamma} y^{\alpha}\right) \partial_{y}^{\beta}\right),
$$

where $\alpha$ and $\beta$ are multi-indices, and, as usual

$$
\begin{aligned}
y^{\alpha} & =y_{1}^{\alpha_{1}} y_{2}^{\alpha_{2}} \ldots y_{d}^{\alpha_{d}}, \\
\partial_{y}^{\beta} & =\left(\frac{\partial}{\partial y_{1}}\right)^{\beta_{1}}\left(\frac{\partial}{\partial y_{2}}\right)^{\beta_{2}} \cdots\left(\frac{\partial}{\partial y_{d}}\right)^{\beta_{d}} .
\end{aligned}
$$

Moreover,

$$
\begin{aligned}
& |\alpha|=\# \text { edges terminating at } \overline{1} \\
& |\beta|=n+l-\# \text { edges teminating at other vertices. }
\end{aligned}
$$

Subtracting second equation from the first, obtain $|\alpha|-|\beta|=n-l$.

We now proceed with the proof of Theorem 0.1 .

LEMMA 2.2. $-\left|A_{n}\right|<(8 \mathrm{e})^{n} n$ !.

Proof. - To describe any graph from $A_{n}$, it suffices to provide the following data for each vertex $j, 1 \leqslant j \leqslant n$ : 
- the source of an incoming edge, if any ( $n$ choices, counting the case when there is no incoming edge);

- whether the vertex is connected to the vertex $\overline{1}$, vertex $\overline{2}$, both or neither (4 choices).

Hence we have at most $4 n$ choices for each of $n$ vertices of the first type. Taking into account the possible labelings of the outgoing edges at each vertex, we get

$$
\left|A_{n}\right| \leqslant 2^{n}(4 n)^{n}
$$

Now the statement follows from the inequality $n^{n}<\mathrm{e}^{n} n$ !.

Lemma 2.3. - Let $\Gamma \in A_{n}$. Then $\left|w_{\Gamma}\right| \leqslant 4^{n}$.

Proof. - Recall that the weight $w_{\Gamma}$ associated to the graph $\Gamma \in A_{n}$ is given by the formula

$$
w_{\Gamma}=\int_{C_{n, 2}^{+}} \omega_{\Gamma},
$$

where $\omega_{\Gamma}$ is the pullback of the volume form from the $2 n$-dimensional torus $\mathbb{T}^{2 n}$ under $\Phi_{\Gamma}$.

We consider the preimage of a generic point $\varphi \in \mathbb{T}^{2 n}$ under $\Phi_{\Gamma}$. Using the action of $G^{1}$, we can fix $q_{1}=0$ and $q_{2}=1$, and thus identify $C_{n, 2}^{+}$with $\mathcal{H}^{n}$.

Rewriting the formula (4) as

$$
\left(z_{2}-z_{1}\right)\left(\bar{z}_{2}-z_{1}\right)=\mathrm{e}^{2 \sqrt{-1} \phi_{e}}\left(z_{2}-\bar{z}_{1}\right)\left(\bar{z}_{2}-\bar{z}_{1}\right)
$$

we see that each edge gives rise to a pair of quadratic equations involving the coordinates of its endpoints. Thus we obtain a system of $2 n$ quadratic equations in $2 n$ real variables (the real and imaginary parts of $\left.p_{1}, p_{2}, \ldots, p_{n}\right)$. Generically, this system has at most $2^{2 n}=4^{n}$ (complex) solutions.

Therefore, we conclude that

$$
\left|w_{\Gamma}\right| \leqslant 4^{n} V
$$

where $V$ is the volume of $\Phi_{\Gamma}\left(C_{n, 2}^{+}\right) \subset \mathbb{T}^{2 n}$. Obviously, $V \leqslant 1$ and the statement follows.

Proof of Theorem 0.1. - Without loss of generality, we may take

$$
p=x_{1}^{a_{1}} x_{2}^{a_{2}} \ldots x_{d}^{a_{d}}, \quad \text { with } a_{1}+\cdots+a_{d}=l .
$$

According to the formulas (6), (7) and Lemma 2.1,

$$
r \star p=r \cdot\left(\sum_{n=0}^{\infty} \frac{1}{n !} \sum_{\Gamma \in A_{n}}^{\infty} w_{\Gamma} \partial_{\Gamma}^{p}\right) .
$$

The expression above can be rewritten as

$$
r \star p=r \cdot\left(\sum_{|\beta| \leqslant l}\left(\sum_{\alpha} c_{\alpha \beta} y^{\alpha}\right) \partial_{y}^{\beta}\right) .
$$

Set $m=l+|\alpha|-|\beta|$. It follows from the discussion after Lemma 2.1, that only the graphs $\Gamma \in A_{m}$ can contribute to $c_{\alpha \beta}$. Observe that all partial derivatives of $\gamma^{i j}$ are at most 1 in absolute 
value (this follows from the inequality (11)), and that any derivative of $p$ is a monomial with the coefficient not exceeding $C_{p}=a_{1} ! a_{2} ! \ldots a_{d} !$. Therefore the absolute value of the coefficient $c_{\alpha \beta}^{\Gamma}$ in (12) does not exceed $C_{p}$.

Using Lemma 2.3, we obtain

$$
\left|c_{\alpha \beta}\right|=\frac{1}{m !}\left|\sum_{\Gamma \in A_{m}} w_{\Gamma} c_{\alpha \beta}^{\Gamma}\right| \leqslant \frac{\left|A_{m}\right|}{m !} 4^{m} C_{p} .
$$

Now by Lemma 2.2, $\left|A_{m}\right|<(8 \mathrm{e})^{m} m$ !, hence

$$
\left|c_{\alpha \beta}\right|<C_{p}(32 \mathrm{e})^{m} \leqslant C_{p}^{\prime}(32 \mathrm{e})^{|\alpha|},
$$

where $C_{p}^{\prime}=(32 \mathrm{e})^{l} C_{p}$.

Therefore, the series $\sum_{\alpha} c_{\alpha \beta} y^{\alpha}$ converges absolutely in the polydisk of radius $1 /(32 \mathrm{e})$. Hence in this neighborhood the formal series (13) defines a differential operator of the form $\sum_{|\beta| \leqslant l} M_{\beta} \partial^{\beta}$, where $M_{\beta}$ is the operator of multiplication by some analytic function. The statement follows.

\section{Tangent map for Lie algebras}

We start by establishing Proposition 0.2.

Proof of Proposition 0.2. - It suffices to show that the constants $c_{2 k}^{(1)}$ from [1, 8.3.3] satisfy estimates of the form

$$
c_{2 k}^{(1)} \leqslant c^{k}
$$

for some constant $c$.

According to [1, 8.3.3.1], we have

$$
c_{n}^{(1)}=\frac{w_{\mathrm{Wh}_{n}}}{2^{n}},
$$

where "the wheel" $\mathrm{Wh}_{n}$ is the graph from $H_{n}$ shown in Fig. 2.

Arguing as in the proof of Lemma 2.3, we conclude that $w_{\mathrm{Wh}_{n}} \leqslant 4^{n}$, hence $c_{n}^{(1)} \leqslant 2^{n}$.

By [1, Th. 8.3.3], if $r \in \mathcal{S}(\mathfrak{g})$ is regarded as a function on $\mathfrak{g}^{*}$, then $I_{T}(r)$ is given by the application of the infinite differential operator associated to the series $S_{1}(x)$ from

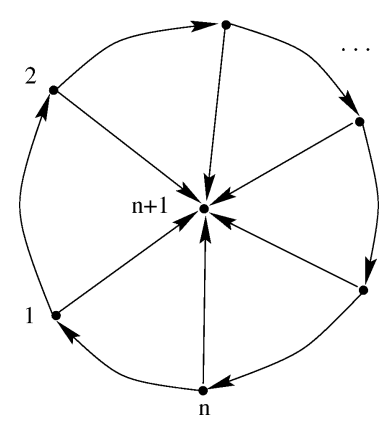

Fig. 2. The wheel $\mathrm{Wh}_{n}$. 


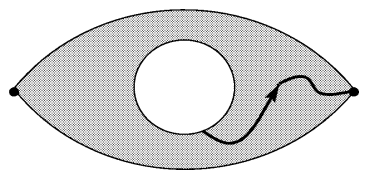

Fig. 3. Path in THE EYE.

Proposition 0.2. Regarding $r$ as a distribution on $\mathfrak{g}$, we conclude that

$$
I_{T}(r)=r \tau .
$$

We can now proceed with the proof of Theorem 0.3.

Let $\Gamma$ be a graph in $A_{n}\left(V_{\Gamma}=\{1,2, \ldots, n, \overline{1}, \overline{2}\}\right)$. For each configuration in $C_{n+2,0}$ (as opposed to $C_{n, 2}$ earlier), we can draw $\Gamma$ in $\mathcal{H}$ and calculate the edge angles. This gives a map

$$
\Phi_{\Gamma}^{\prime}: \bar{C}_{n+2,0} \rightarrow \mathbb{T}^{2 n}
$$

and we denote by $\omega_{\Gamma}^{\prime}$ the pullback of the volume form on the torus.

Next recall that $\bar{C}_{2,0}$ (the EYE) is the compactified configuration space of two points in $\mathcal{H}$. We fix a path $\xi:[0,1] \rightarrow \bar{C}_{2,0}$, such that

$$
\begin{aligned}
& \xi(0) \in C_{2} \quad \text { (two points coinciding in } \mathcal{H} \text { ), } \\
& \xi(1) \in C_{0,2}^{+} \quad \text { (both points lying in } \mathbb{R} \subset \overline{\mathcal{H}} \text { ). }
\end{aligned}
$$

Now for each $n \geqslant 0$ we have a "forgetting" map $\bar{C}_{n+2,0} \rightarrow \bar{C}_{2,0}$, where we forget the location of first $n$ points in the configuration. We denote by $Y_{n} \subset \bar{C}_{n+2,0}$ the preimage of the path $\xi([0,1])$ under the forgetting map and by $\partial Y_{n}$ its boundary.

The boundary $\partial Y_{n}$ can be written as the following cycle:

$$
\partial Y_{n}=Z_{n}^{0}-Z_{n}^{1}-Z_{n}
$$

where $Z_{n}^{0}, Z_{n}^{1}$ are the preimages of the points $\xi(0)$ and $\xi(1)$ respectively and $Z_{n}$ is the sum (with appropriate signs) of the components of

$$
\partial \bar{C}_{n+2,0} \cap \text { preimage of }\{\xi(t): 0<t<1\} .
$$

Since $\omega_{\Gamma}^{\prime}$ is a closed $2 n$-form, by Stokes' theorem we get

$$
\int_{Z_{n}^{0}} \omega_{\Gamma}^{\prime}-\int_{Z_{n}^{1}} \omega_{\Gamma}^{\prime}=\int_{Z_{n}} \omega_{\Gamma}^{\prime} .
$$

Proof of Theorem 0.3. - By [1, 8.2.1, 8.2.2], for $r, p \in \mathcal{S}$ we have

$$
\begin{aligned}
& r \cdot\left[\sum_{n=0}^{\infty} \frac{1}{n !} \sum_{\Gamma \in A_{n}} \partial_{\Gamma}^{p}\left(\int_{Z_{n}^{1}} \omega_{\Gamma}^{\prime}\right)\right]=(r \tau) \star(p \tau), \\
& r \cdot\left[\sum_{n=0}^{\infty} \frac{1}{n !} \sum_{\Gamma \in A_{n}} \partial_{\Gamma}^{p}\left(\int_{Z_{n}^{0}} \omega_{\Gamma}^{\prime}\right)\right]=\left(r *_{\mathfrak{g}} p\right) \tau .
\end{aligned}
$$

Hence for $T_{p}$ as in the statement of Theorem 0.3 , we get 


$$
r \cdot T_{p}=\left(r *_{\mathfrak{g}} p\right) \tau-(r \tau) \star(p \tau)=r \cdot\left[\sum_{n=0}^{\infty} \frac{1}{n !} \sum_{\Gamma \in A_{n}} \partial_{\Gamma}^{p}\left(\int_{Z_{n}} \omega_{\Gamma}^{\prime}\right)\right] .
$$

We now discuss the structure of $Z_{n}$. The relative positions of the vertices $\overline{1}$ and $\overline{2}$ are determined by the point $\xi(t) \in C_{2,0}$. The integral $\int_{Z_{n}} \omega_{\Gamma}^{\prime}$ is the sum of integrals over the top (i.e., $2 n$-dimensional) components of $Z_{n}$, which correspond to the following (degenerate) configurations:

1. Two or more points cluster at $\overline{1}$.

2. Two or more points cluster at $\overline{2}$.

3. Two or more points cluster somewhere else in $\mathcal{H}$.

4. One or more points cluster on $\mathbb{R}$.

For components of type $4 \omega_{\Gamma}^{\prime}=0$ (since the angle for any edge originating from $\mathbb{R}$ is identically 0 ). For components of types 1-3 which involve clusters of three or more points, $\int \omega_{\Gamma}^{\prime}=0$ by [1, Lemma 6.6].

Now let $Z$ be a component corresponding to a two-point cluster of type 3 . The corresponding boundary component of $\bar{C}_{n+2,0}$ is $C_{2} \times C_{n+1,0}$ and we have $Z \simeq C_{2} \times \widetilde{Z}$, where $\widetilde{Z}$ is the preimage of the path $\xi$ in $C_{n+1,0}$ under the forgetting map. As in [1, 6.4.1], $\int_{Z} \omega_{\Gamma}^{\prime}$ decomposes as a product of integrals over $C_{2}$ and $\widetilde{Z}$. The first integral vanishes unless two points in the cluster are connected by an edge.

$$
\cdots \stackrel{k}{\longleftarrow} \stackrel{\gamma^{k l} \stackrel{l}{\bullet} \gamma^{i j}}{\longrightarrow} \underset{j}{\stackrel{i}{\rightrightarrows}} \cdots .
$$

Relabeling the vertices and edges as above, we see that the operator $\partial_{\Gamma}^{p}$ is a sum of terms with factors of the form

$$
\sum_{l=1}^{d} \gamma^{k l} \frac{\partial \gamma^{i j}}{\partial x_{l}}=\frac{1}{4} \sum_{l=1}^{d}\left[x_{k}, x_{l}\right] c_{i j}^{l}=\frac{1}{4}\left[x_{k},\left[x_{i}, x_{j}\right]\right]
$$

The set of graphs with the above subgraph can be grouped into sets of three corresponding to a cyclic permutation of the three outgoing edges from the cluster. Each of these graphs has the same weight $\int_{Z} \omega_{\Gamma}^{\prime}$. By the Jacobi identity, the sum of the three corresponding operators $\partial_{\Gamma}^{p}$ vanishes. Hence $\sum_{\Gamma \in A_{n}} \partial_{\Gamma}^{p}\left(\int_{Z} \omega_{\Gamma}^{\prime}\right)=0$.

Now we consider two-point clusters of type 2 .

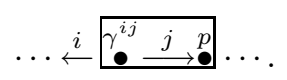

Relabeling the edges and vertices as above, we see that the corresponding operator is a sum of terms with factors of the form

$$
\sum_{j=1}^{d}\left(p \cdot y_{j}\right)\left[x_{i}, x_{j}\right]
$$

where $\left[x_{i}, x_{j}\right] \in \mathfrak{g}$ acts as a differential operator. But this expression is equal to $p \cdot \operatorname{adj}_{x_{i}}$ (where $\operatorname{adj}_{x_{i}}$ is the adjoint vector field corresponding to $x_{i} \in \mathfrak{g}$ ), which vanishes since $p$ is invariant $(p \in \mathcal{I})$.

This leaves only two-point clusters of type 1 .

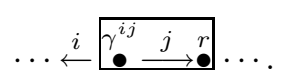


Arguing as above, we conclude that $r \cdot T_{p}$ is a sum of terms containing factors of the type $r \cdot \operatorname{adj} j_{x_{i}}$. Hence $T_{p}$ is an infinite sum of operators from $\mathfrak{R}$. By Theorem 0.1 and Proposition 0.2

$$
T_{p}=\partial_{p} \tau-\tau \partial_{p \tau}^{\star}
$$

belongs to $\mathfrak{D}$. Therefore, $T_{p} \in \mathfrak{R}$, as was to be shown.

Remarks. - (a) In the above discussion we did not explicitly take into consideration the possibility of edges originating outside the cluster and terminating at the vertices of the cluster. However, we can group together graphs which differ only by a permutation of the incident vertices in the cluster, and observe that these graphs have the same weight. The result now follows from the Leibniz formula.

(b) The components of $\partial Y_{n}$ have two natural orientations: the first arising from their expression as products of configuration spaces and the second inherited from $Y_{n}$. Strictly speaking, one should check that these orientations are "compatible" in the sense that the formulas (14)-(16) indeed hold as asserted.

The question of signs in formulas (15), (16) goes to the heart of the definition of the Kontsevich star-product. These signs are not discussed in $[1,8.2 .1,8.2 .2]$, but can be checked along the lines of [3]. As for formula (14), clearly the sign of the components of $Z_{n}$ is irrelevant. We need only show that the terms on the left occur with opposite signs. Since these signs are "universal", i.e. independent of the Lie algebra, we consider the case of an abelian Lie algebra $\mathfrak{g}$. Then $\tau=1$ and $r \star p=r *_{\mathfrak{g}} p$ for any $r, p$ in $S(\mathfrak{g})$. Hence the expressions (15), (16) can enter (14) with opposite signs only.

\section{Kashiwara-Vergne conjecture and local solvability}

In this section we prove the Kashiwara-Vergne conjecture (Theorem 0.4) and discuss its consequences.

We start by observing, that for $x^{\prime}$ and $x^{\prime \prime}$ in $\mathfrak{g}$, we have

$$
\left[x^{\prime}, x^{\prime \prime}\right]=x^{\prime} \star x^{\prime \prime}-x^{\prime \prime} \star x^{\prime} .
$$

Then, by the universal property of $\mathcal{U}=\mathcal{U}(\mathfrak{g})$ it follows that there is a unique algebra homomorphism between $\mathcal{U}$ and $(\mathcal{S}, \star)$. This morphism (denoted $I_{\text {alg }}$ in $[1,8.3 .1]$ ) is invertible, and we shall denote its inverse by $\kappa$.

Then, as established in $[1,8.3 .4]$, we have

$$
\kappa(p \tau)=\exp _{*}(p q)=\eta(p) \quad \text { for } p \in \mathcal{S} .
$$

Proof of Theorem 0.4. - In view of Theorem 0.3, it suffices to establish the following identity (in $\mathfrak{D})$

$$
D_{p}=T_{p} \tau^{-1} q .
$$

Since both sides are germs of analytic differential operators, it is enough to verify that

$$
r \cdot D_{p}=\left(r \cdot T_{p}\right) \tau^{-1} q
$$

for all $r \in \mathcal{S}$. 
Then

$$
\begin{aligned}
r \cdot D_{p} & =\exp ^{*}\left[\eta\left(r *_{\mathfrak{g}} p\right)-\eta(r) *_{G} \eta(p)\right] \\
& =\left(r *_{\mathfrak{g}} p\right) q-\exp ^{*}\left(\kappa(r \tau) *_{G} \kappa(p \tau)\right) \\
& =\left(r *_{\mathfrak{g}} p\right) q-\exp ^{*}(\kappa(r \tau \star p \tau)) \\
& =\left(r *_{\mathfrak{g}} p\right) q-\exp ^{*}\left(\eta\left((r \tau \star p \tau) \tau^{-1}\right)\right) \\
& =\left(r *_{\mathfrak{g}} p\right) q-(r \tau \star p \tau) \tau^{-1} q \\
& =\left[\left(r *_{\mathfrak{g}} p\right) \tau-(r \tau \star p \tau)\right] \tau^{-1} q \\
& =\left(r \cdot T_{p}\right) \tau^{-1} q .
\end{aligned}
$$

Theorems 0.5 and 0.6 follow immediately from the theorem above, and Theorem 0.7 requires a short argument, which we give below.

Proof of Theorem 0.7. - A bi-invariant differential operator on $G$ is precisely an element of the center $\mathcal{Z}$ of $\mathcal{U}$, hence of the form $\eta(p)$ for some $p \in \mathcal{I}$. Let $\delta_{0}$ and $\delta_{1}$ be the delta-distributions supported at $0 \in \mathfrak{g}$ and $1 \in G$ respectively. Then if $p \in \mathcal{I} \backslash\{0\}$, by [20] (see also [21]), there exists a germ $P \in \mathbf{I}$, such that

$$
P *_{\mathfrak{g}} p=\delta_{0}
$$

Hence by Theorem 0.5 , we get

$$
\eta(P) *_{G} \eta(p)=\eta\left(\delta_{0}\right)=\delta_{1},
$$

and we see that $\eta(P)$ is a local fundamental solution for $\eta(p)$.

\section{Results for Lie supergroups}

\subsection{Preliminaries on supermanifolds}

We briefly discuss basic facts about supermanifolds. The study of supermanifolds etc. was initiated by Berezin (see [4,19]) and independently by Kostant [17]. Subsequently a slightly different (though essentially equivalent) treatment appeared in the physics literature [5]. We will follow the approach of [17]. The reader familiar with the physics point of view should have no trouble translating everything into that language.

A supermanifold $\left(\mathbb{Z}_{2}\right.$-graded manifold) of dimension $\left(d_{0}, d_{1}\right)$ is a triple $(X, \mathcal{A}, \pi)$, where $X$ is a $d_{0}$-dimensional smooth manifold, $\mathcal{A}$ is a sheaf of $\mathbb{Z}_{2}$-graded commutative algebras and $\pi$ is a sheaf map from $\mathcal{A}$ to the sheaf $\mathcal{C}^{\infty}(X)$ of smooth functions on $X$. Moreover, we require that every open subset of $X$ can be covered by " $\mathcal{A}$-splitting open sets $U$ of odd dimension $d_{1}$ ". This means that we can choose (non-canonical) subalgebras $C(U)$ and $D(U)$ of $\mathcal{A}(U)$ such that

$$
\begin{aligned}
& \left.\pi\right|_{C(U)}: C(U) \longrightarrow C^{\infty}(U) \text { is an algebra isomorphism, } \\
& D(U) \text { is isomorphic to the exterior algebra in } d_{1} \text { variables, } \\
& C(U) \otimes D(U) \simeq \mathcal{A}(U) \text { (algebra isomorphism). }
\end{aligned}
$$

$\mathcal{A}(X)$ and its dual $\mathcal{A}(X)^{\prime}=\operatorname{Hom}_{\mathbb{R}}(\mathcal{A}(X), \mathbb{R})$ are super (i.e., $\mathbb{Z}_{2}$-graded) vector spaces. A "pure" linear functional $v \in \mathcal{A}(X)^{\prime}$ is called a differentiation at $x \in X$ if

$$
\langle v, f g\rangle=\langle v, f\rangle \widetilde{g}(x)+(-1)^{p(v) p(f)} \widetilde{f}(x)\langle v, g\rangle,
$$


for any pure $f$ and $g$ in $\mathcal{A}(X)$. Here we write $\tilde{f}$ for $\pi(f)$ and $p$ for the parity ( 0 or 1$)$. An arbitrary $v \in \mathcal{A}(X)^{\prime}$ is called a differentiation if its homogeneous components are differentiations, and we write $T_{x}(X, \mathcal{A})$ for the super vector space of all differentiations at $x$. It is easy to see that $\operatorname{dim}\left[T_{x}(X, \mathcal{A})\right]_{i}=d_{i}, i=0,1$. Moreover, we have a natural surjection $\pi_{x}: T_{x}(X, \mathcal{A}) \longrightarrow T_{x}(X)$ (ordinary tangent space of $X$ at $x$ ) with $\operatorname{ker} \pi_{x}=\left[T_{x}(X, \mathcal{A})\right]_{1}$.

For each $k=0,1, \ldots$ the sheaf $\operatorname{Diff}_{k} \mathcal{A}$ of differential operators of order $\leqslant k$ on the supermanifold $(X, \mathcal{A})$ is defined in $[17,2.10]$ as follows:

$$
\begin{aligned}
& \left(\operatorname{Diff}_{0} \mathcal{A}\right)(U)=\mathcal{A}(U), \text { regarded as left multiplication operators; } \\
& \left(\operatorname{Diff}_{k} \mathcal{A}\right)(U)=\left\{\partial \in \operatorname{End} \mathcal{A}(U):[\partial, f] \in\left(\operatorname{Diff}_{k-1} \mathcal{A}\right)(U) \text { for all } f \in \mathcal{A}(U)\right\} .
\end{aligned}
$$

Here the bracket $[$,$] denotes the graded commutator in \operatorname{End} \mathcal{A}(U)$.

For $\partial \in\left(\operatorname{Diff}_{k} \mathcal{A}\right)(X)$ we can define its body as the unique differential operator $\widetilde{\partial}$ on $X$ satisfying the conditions

$$
\begin{aligned}
\widetilde{\partial} \cdot 1_{C^{\infty}(X)} & =\widetilde{\partial \cdot 1_{\mathcal{A}}} \text { and } \\
{[\widetilde{\partial}, \widetilde{f}] } & =\widetilde{[\partial, f]} \text { for all } f \in \mathcal{A}(X) .
\end{aligned}
$$

This allows us to introduce a locally convex topology on $\mathcal{A}(X)$, as in [17, 2.9]. The space of distributions $\operatorname{Dist}(X, \mathcal{A})$ can then be defined as the topological dual of $\mathcal{A}(X)$. The support of a function $f \in \mathcal{A}(X)$ is simply the support of $\pi(f) \in C^{\infty}(X)$, and the support of a distribution $T \in \operatorname{Dist}(X, \mathcal{A})$ is the smallest closed set $\operatorname{supp} T$ such that $T$ vanishes on all $f \in \mathcal{A}(X)$ with $\operatorname{supp} f \cap \operatorname{supp} T=\emptyset$.

\subsection{Preliminaries on Lie supergroups}

Following [17, 2.11], we define $\mathcal{A}(X)^{*} \subset \mathcal{A}(X)^{\prime}$ as the subset of those linear functionals on $\mathcal{A}(X)$ whose kernel contains an ideal of finite codimension. It is easy to check that $\mathcal{A}(X)^{*}$ is a graded co-commutative coalgebra. We say that the supermanifold $(G, \mathcal{A}, \pi)$ is a Lie supergroup (graded Lie group in [17]), if $\mathcal{A}(G)^{*}$ has the structure of a Hopf algebra extending the natural coalgebra structure.

This implies that $G$ is a Lie group and that the super tangent space $\mathfrak{g}=T_{e}(G, \mathcal{A})$ at the identity $e \in G$ has the structure of a Lie superalgebra, i.e.,

$$
\mathfrak{g}=\mathfrak{g}_{0}+\mathfrak{g}_{1},
$$

where $\mathfrak{g}_{0}$ is the Lie algebra of $G$. Moreover, we have a representation $\pi$ of $G$ on $\mathfrak{g}_{1}$, whose differential is the adjoint action of $\mathfrak{g}_{0}$ on $\mathfrak{g}_{1}$. We have used the same letter $\pi$ to emphasize the fact that the supergroup can be reconstructed from the representation. The Hopf algebra $\mathcal{A}(G)^{*}$ can be obtained as the smash product of the group algebra $\mathbb{R} G$ and the universal enveloping algebra $\mathcal{U}(\mathfrak{g})$, and then $\mathcal{A}(G)$ can be recovered as in [17, 3.7].

The action of a Lie supergroup $(G, \mathcal{A})$ on a supermanifold is defined in [17, 3.9]. Each Lie supergroup $(G, \mathcal{A})$ has an "adjoint action" on itself and on its Lie superalgebra $\mathfrak{g}$ (regarded as a supermanifold). Thus for each $a \in \mathfrak{g}$ we obtain derivations of the algebras $\mathcal{A}(G)$ and $\mathcal{A}(\mathfrak{g})$, which will both be denoted by $\operatorname{adj}_{a}$.

As before, we regard the (graded) symmetric algebra $\mathcal{S}=\mathcal{S}(\mathfrak{g})$ as the convolution algebra of distributions on the super vector space $\mathfrak{g}$ with support at $0 \in \mathfrak{g}$ and denote by $\mathcal{I}$ the subalgebra of adj-invariant distributions.

We regard the universal enveloping algebra $\mathcal{U}=\mathcal{U}(\mathfrak{g})$ as the convolution algebra of distributions on the Lie supergroup $(G, \mathcal{A}, \pi)$ with support at $e \in G$. The center $\mathcal{Z}$ of $\mathcal{U}$ can then be regarded as the subalgebra of adj-invariant distributions. 
The spaces of germs $\mathbf{S}, \mathbf{I}, \mathbf{U}, \mathbf{Z}$ (see Introduction) can be defined analogously in the supersetting.

The exponential map from $\mathfrak{g}$ to $(G, \mathcal{A})$ is best described in terms of the corresponding "pushforward" map

$$
\exp _{*}: \mathcal{A}(\mathfrak{g})^{*} \longrightarrow \mathcal{A}(G)^{*}
$$

which is the usual exponential from $\mathbb{R} \mathfrak{g}$ to the group algebra $\mathbb{R} G$, and the symmetrization map from $\mathcal{S}$ to $\mathcal{U}$.

At the germ level $\exp _{*}$ is an isomorphism from $\mathbf{S}$ to $\mathbf{U}$ (and $\mathbf{I}$ to $\mathbf{Z}$ ). Hence it has a well-defined inverse exp*.

As in the Introduction, we denote by $\mathfrak{D}$ the algebra of germs at 0 of differential operators on $\mathfrak{g}$ with analytic coefficients, and by $\mathfrak{R}$ the right ideal of $\mathfrak{D}$ generated by the germs of the differential operators $\operatorname{adj}_{a}, a \in \mathfrak{g}$.

\subsection{Statements and proofs for Lie supergroups}

All results stated in Sections 0-4 hold with minor changes in the statements and proofs. We discuss the less obvious modifications below.

Let $\mathfrak{g}$ be a finite-dimensional real Lie superalgebra. Then its dual $\mathfrak{g}^{*}=\operatorname{Hom}_{\mathbb{R}}(\mathfrak{g}, \mathbb{R})=\mathfrak{g}_{0}^{*}+\mathfrak{g}_{1}^{*}$ is a Poisson supermanifold (cf. [17, 5.2]). The Poisson bracket $\gamma$ defined as the unique biderivation of $\mathcal{A}\left(\mathfrak{g}^{*}\right)$ satisfying

$$
\gamma\left(f_{1}, f_{2}\right)= \begin{cases}0 & \text { if } f_{1} \text { or } f_{2} \text { is constant, } \\ \frac{1}{2}\left[f_{1}, f_{2}\right] & \text { if } f_{1}, f_{2} \in \mathfrak{g} .\end{cases}
$$

For convenience we fix bases $x_{1}, x_{2}, \ldots, x_{d_{0}}$ of $\mathfrak{g}_{0}$ and $x_{d_{0}+1}, \ldots, x_{d_{0}+d_{1}}$ of $\mathfrak{g}_{1}$. Then in coordinates we have

$$
\gamma\left(f_{1}, f_{2}\right)=\frac{1}{2} \sum_{i, j}\left[x_{i}, x_{j}\right] \frac{\partial f_{1}}{\partial x_{i}} \frac{\partial f_{2}}{\partial x_{j}} .
$$

For an odd variable $x_{i}$ the vector field $\frac{\partial}{\partial x_{i}}$ is odd; nevertheless, $\gamma$ is an even bidifferential operator and preserves the grading on $\mathcal{A}\left(\mathfrak{g}^{*}\right)$.

Following [1], we can define the $\star$-product of $f_{1}, f_{2} \in \mathcal{A}\left(\mathfrak{g}^{*}\right)$ by the formula

$$
f_{1} \star f_{2}=\sum_{n=0}^{\infty} \frac{\hbar^{n}}{n !} \sum_{\Gamma \in G_{n}} w_{\Gamma} B_{\Gamma}\left(f_{1}, f_{2}\right) \text {. }
$$

The bidifferential operators $B_{\Gamma}$ for $\Gamma \in G_{n}$ are given by the formula (8) up to a sign which can be calculated in the following way (illustrated by the example below).

We consider the same graph $\Gamma_{0} \in G_{2}$ as in Section 1.2, i.e.

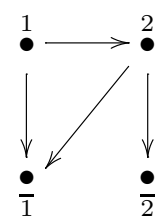

or (relabeling)

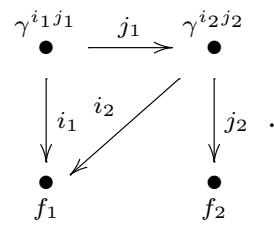


We first form the expression

$$
\sum_{1 \leqslant i_{1}, j_{1}, i_{2}, j_{2} \leqslant d}\left(\partial_{i_{1}} \gamma^{i_{1} j_{1}} \partial_{j_{1}}\right)\left(\partial_{i_{2}} \gamma^{i_{2} j_{2}} \partial_{j_{2}}\right) f_{1} f_{2}
$$

where the bivector fields $(\gamma)$ corresponding to the vertices 1 and 2 are all written on the left (since $\gamma$ is even, the order is irrelevant). Then we use the Koszul rule of signs to move the derivatives to the appropriate positions (as given by the graph), i.e. $\partial_{i_{1}}$ and $\partial_{i_{2}}$ should be moved to the left of $f_{1}, \partial_{j_{2}}$ to $f_{2}$ and $\partial_{j_{1}}$ to $\gamma^{i_{2} j_{2}}$. We obtain the bidifferential operator, as in the formula (9) before

$$
\sum_{1 \leqslant i_{1}, j_{1}, i_{2}, j_{2} \leqslant d} \pm \gamma^{i_{1} j_{1}} \partial_{j_{1}}\left(\gamma^{i_{2} j_{2}}\right) \partial_{i_{1}} \partial_{i_{2}}\left(f_{1}\right) \partial_{j_{2}}\left(f_{2}\right) .
$$

Here the signs of the summands are given by -1 to the power

$$
p\left(j_{2}\right) p\left(f_{1}\right)+p\left(i_{2}\right)\left(p\left(i_{2}\right)+p\left(j_{2}\right)\right)+p\left(i_{1}\right)\left(p\left(i_{1}\right)+p\left(i_{2}\right)+p\left(j_{2}\right)\right),
$$

where $p(i)$ is the parity of $x_{i} \in \mathfrak{g}$ and $p(f)$ is the parity of the $f \in \mathcal{A}\left(\mathfrak{g}^{*}\right)$. Note that in the above formula (18) we did not move $\partial_{i_{1}}$ past $\partial_{i_{2}}$, thus preserving the same order as in (17).

It is easy to check that Kontsevich's argument for the associativity of the $\star$-product remains valid for $\mathcal{A}\left(\mathfrak{g}^{*}\right)$.

Proceeding as in Section 2, we obtain

THEOREM 5.1. - Given $p \in \mathcal{S}$ of order $l$, there is a unique element $\partial_{p}^{\star}$ of order $l$ in $\mathfrak{D}$ such that for all $r \in \mathcal{S}$

$$
r \star p=r \cdot \partial_{p}^{\star} .
$$

The analyticity of the coefficients of $\partial_{p}^{\star}$ is verified exactly as before.

Let us write str for the supertrace of an endomorphism of a graded vector space [19]. The expression $\operatorname{str}\left[(\operatorname{ad} x)^{2 k}\right]$ defines an even element in $\mathcal{A}(\mathfrak{g})$. With $c_{2 k}^{(1)}$ as in (1), we can verify (as in Section 3) that

$$
S_{1}(x)=\exp \left(\sum_{k=1}^{\infty} c_{2 k}^{(1)} \operatorname{str}\left[(\operatorname{ad} x)^{2 k}\right]\right)
$$

converges to an even superfunction $\tau(x)$ in $\mathcal{A}(\mathfrak{g})$ which is analytic at $0 \in \mathfrak{g}$.

Proceeding as in Section 3, we obtain

THEOREM 5.2.- For $p \in I$, the operator

$$
T_{p}=\partial_{p} \tau-\tau \partial_{p \tau}^{\star}
$$

belongs to $\Re$.

Just as for $\tau(x)$, the expression

$$
q(x)=\exp \left(\sum_{k=1}^{\infty} \frac{B_{2 k}}{4 k(2 k) !} \operatorname{str}\left[(\operatorname{ad} x)^{2 k}\right]\right)
$$

defines an element in $\mathcal{A}(\mathfrak{g})$, which is analytic on $\mathfrak{g}$. 
The map $\eta: \mathbf{S} \longrightarrow \mathbf{U}$ is defined (as before) by

$$
\eta(p)=\exp _{*}(p q)
$$

and the restriction of $\eta$ to $\mathcal{I}$ is an algebra isomorphism from $\mathcal{I}$ to $\mathcal{Z}$ (this extension of Duflo's isomorphism to the case of Lie superalgebras is due to Kontsevich).

Theorems $0.4,0.5$ and 0.6 for any real Lie superalgebra $\mathfrak{g}$ now follow from Theorem 5.2, as in Section 4.

The statement of Theorem 0.7 should be modified in the following manner:

THEOREM 5.3. - Any bi-invariant differential operator on a real Lie supergroup G, with a nonzero body, admits a (local) fundamental solution and hence is locally solvable.

Proof. - If a bi-invariant differential operator on a real Lie supergroup $G$ has a nonzero body, it can be represented as $\eta(p)$, where $p \in \mathcal{I}$ and $p$ has a nonzero body. Then we can find a germ $P \in \mathbf{I}$, such that

$$
P *_{\mathfrak{g}} p=\delta_{0} .
$$

Local solvability of $\eta(p)$ now follows as in the proof of Theorem 0.7 .

\section{Acknowledgements}

We would like to thank M. Kontsevich for sharing his insights with us and P. Deligne for many valuable suggestions. We also thank the participants of the Gelfand seminar at Rutgers in which we first learned the main ideas behind the Kontsevich formality theorem. Finally, we thank M. Duflo and D. Manchon for useful correspondence at an early stage of this project. This work began in the spring and summer of 1998, when the first author was visiting the Institute for Advanced Study and Rutgers University, and the second author was a Hill Assistant Professor at Rutgers University. Both authors thank the respective institutions for their suppport.

\section{REFERENCES}

[1] Kontsevich M., Deformation quantization of Poisson manifolds I, e-print, math.QA/9709040.

[2] Andler M., Dvorsky A., SAhi S., Deformation quantization and invariant distributions, $C$. $R$. Acad. Sci. 330 (2000) 115-120.

[3] Arnal D., Manchon D., Masmoudi M., Choix des signes pour la formalité de M. Kontsevich, Pacific J. Math. 203 (2002) 23-66.

[4] BereZIN F., The Method of Second Quantization, Academic Press, New York, 1966.

[5] DeWitT B., Supermanifolds, 2nd edition, Cambridge Univ. Press, Cambridge, 1992.

[6] DiXMIER J., Algèbres enveloppantes, Gauthier-Villars, Paris, 1974.

[7] Duflo M., Caractères des algèbres de Lie résolubles, C. R. Acad. Sci. 269 (1969) 437-438.

[8] Duflo M., Sur les extensions des représentations irréductibles des groupes de Lie nilpotents, Ann. Sci. École Norm. Sup. 5 (1972) 71-120.

[9] Duflo M., Construction of primitive ideals in an enveloping algebra, in: Gelfand I. (Ed.), Lie Groups and their Representations, Adam Hilger Ltd, London, 1975.

[10] Duflo M., Opérateurs différentiels bi-invariants sur un groupe de Lie, Ann. Sci. École Norm. Sup. 10 (1977) 267-288.

[11] Duflo M., RaIs M., Sur l'analyse harmonique sur les groupes de Lie résolubles, Ann. Sci. École Norm. Sup. 9 (1976) 107-114.

[12] Fulton W., MacPherson R., Compactification of configuration spaces, Ann. Math. (2) 139 (1994) $183-225$. 
[13] Helgason S., The surjectivity of invariant differential operators on a symmetric space, Ann. Math. (2) 98 (1973) 451-479.

[14] Kashiwara M., Vergne M., The Campbell-Hausdorff formula and invariant hyperfunctions, Invent. Math. 47 (1978) 249-272.

[15] Kirillov A.A., Merits and demerits of the orbit method, Bull. Amer. Math. Soc. 36 (1999) 433-488.

[16] KonTSEVICH M., private communication.

[17] Kostant B., Graded Manifolds, Graded Lie Theory and Prequantization, Lect. Notes in Math., Vol. 570, Springer, Berlin, 1977.

[18] LEWY H., An example of a smooth linear partial differential equation without solution, Ann. Math. (2) 66 (1957) 155-158.

[19] Manin Yu., Gauge Field Theory and Complex Geometry, Springer, Berlin, 1988.

[20] RaIS M., Solutions élémentaires des opérateurs différentiels bi-invariants sur un groupe de Lie nilpotent, C. R. Acad. Sci. 273 (1971) 495-498.

[21] RaIs M., Opérateurs différentiels bi-invariants (d'après M. Duflo), Lect. Notes in Math., Vol. 667, Springer, Berlin, 1978, pp. 125-137.

[22] SHOIKHET B., Vanishing of the Kontsevich integral of the wheels, e-print, math.QA/0007080.

(Manuscript received October 19, 2000;

accepted, after revision, May 4, 2001.)

\author{
Martin ANDLER \\ Département de Mathématiques, \\ Université de Versailles-Saint Quentin, \\ 78035 Versailles Cédex, France
Mathematics Department,
University of Miami, \\ Alexander DVORSKY \\ Coral Gables, FL 33124, USA
}

Siddhartha SAHI

Department of Mathematics,

Rutgers University,

New Brunswick, NJ 08903, USA 